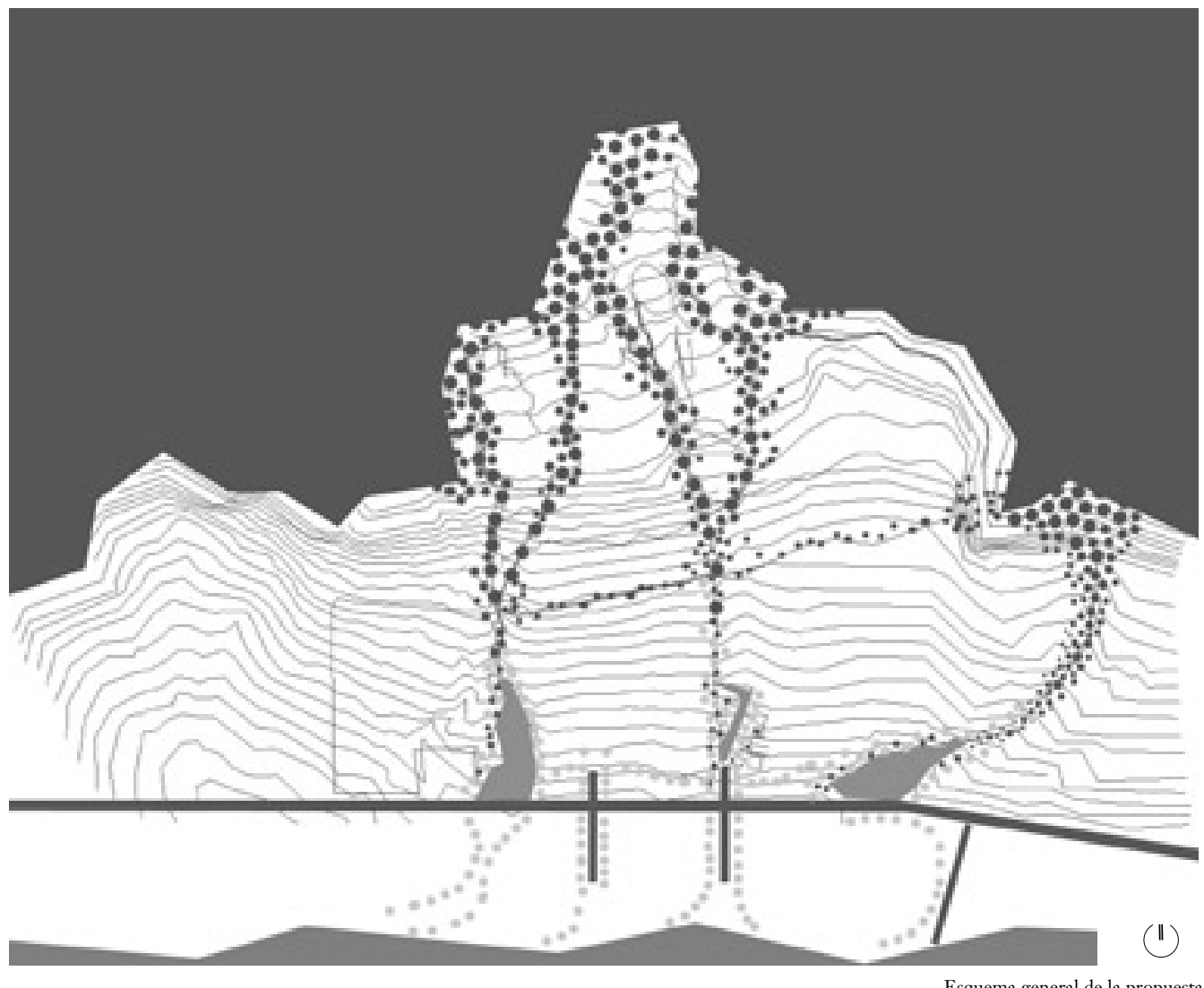

\section{Teodoro Fernández}

Tanto la arquitectura como la arquitectura del paisaje operan a partir de intervenciones que alteran el orden original. En este caso, y por el contrario, se trata de la recuperación de una condición natural perdida: cómo defender la diversidad biológica dentro de una gran superficie de monocultivo. Lo interesante es que a partir de esta reconstitución se revaloriza la estructura espacial de un valle, a partir de su topografía y la manera en que el agua se desplaza por ella.

Palabras clave: Arquitectura del paisaje-Chile, cultivos biodinámicos, cultivos ecológicos, viñas orgánicas.

Architecture -and landscape architecture-operate on the basis of interventions, which seek, one way or another, to change the existing order. In this case, rather than proposing a new order this is an attempt to recover a lost structure, to defend biological diversity within the expanse of a monoculture. Interestingly, the effect of the reconstitution is to re-value the spatial structure of a valley, starting from its topography and the way the water moves through it.

Key words: Landscape architecture-Chile, biodynamic crops, ecological crops,

\title{
Viñas y corredores ecológicos
}

El proyecto de corredores ecológicos para el Fundo Los Robles de los viñedos orgánicos Emiliana en Nancagua, provincia de Colchagua, se realizó con el fin de contribuir a adecuar el área de la viña a un cultivo biodinámico, y se encuentra actualmente en ejecución.

\section{La agricultura biodinámica}

La agricultura biodinámica engloba en un sistema de trabajo las tres finalidades de toda agricultura: la fertilidad de la tierra, la resistencia de las plantas y la calidad de los alimentos producidos, comprendidos como una totalidad. Los principales puntos del método biodinámico fueron expuestos por Rudolf Steiner en un curso para agricultores dado en 1924; desde entonces se han recogido experiencias y llevado a cabo numerosos trabajos. Para que una planta pueda crecer y desarrollarse de forma sana necesita una tierra viva y fértil, capaz de regularse a sí misma y adaptarse a diferentes circunstancias; los fertilizantes químicos actúan de manera directa y exclusiva en las plantas y no mejoran la fertilidad de la tierra. La agricultura biodinámica considera a la tierra como un organismo vivo, que como tal ha de alimentarse y desarrollarse. Además del compost, principal alimento de la tierra, se han desarrollado medios y preparados que sirven para armonizar y regular los procesos en diferentes situaciones: el agua de ortiga, la cola de caballo o los preparados biodinámicos.

\section{Lo importante es la totalidad}

Una viña es en sí un monocultivo de tamaño considerable; en el presente caso del fundo Los Robles se trata de 120 ha. Un monocultivo es naturalmente muy inestable, contrariamente a un bosque o espacio natural que tienden a una gran estabilidad. Por su tamaño los monocultivos tienden a formar barreras o fronteras entre los espacios naturales originales, y cuando abarcan regiones completas ellos pueden quedar convertidos en islas segregadas. Los corredores tratan de unir estas islas, al mismo tiempo que introducen la diversidad y variedad de la naturaleza en las extensiones de monocultivos, transformando el trabajo agrícola de la viña en una agricultura no especializada, sino más bien preocupada de un gran ámbito de la producción de alimentos, del vino, y de otras plantas y los animales.

Los corredores restauran en el predio -haciéndolos visibles- los elementos naturales del paisaje: la topografía, el ciclo del agua y las plantas, uniendo a través de la viña los espacios 


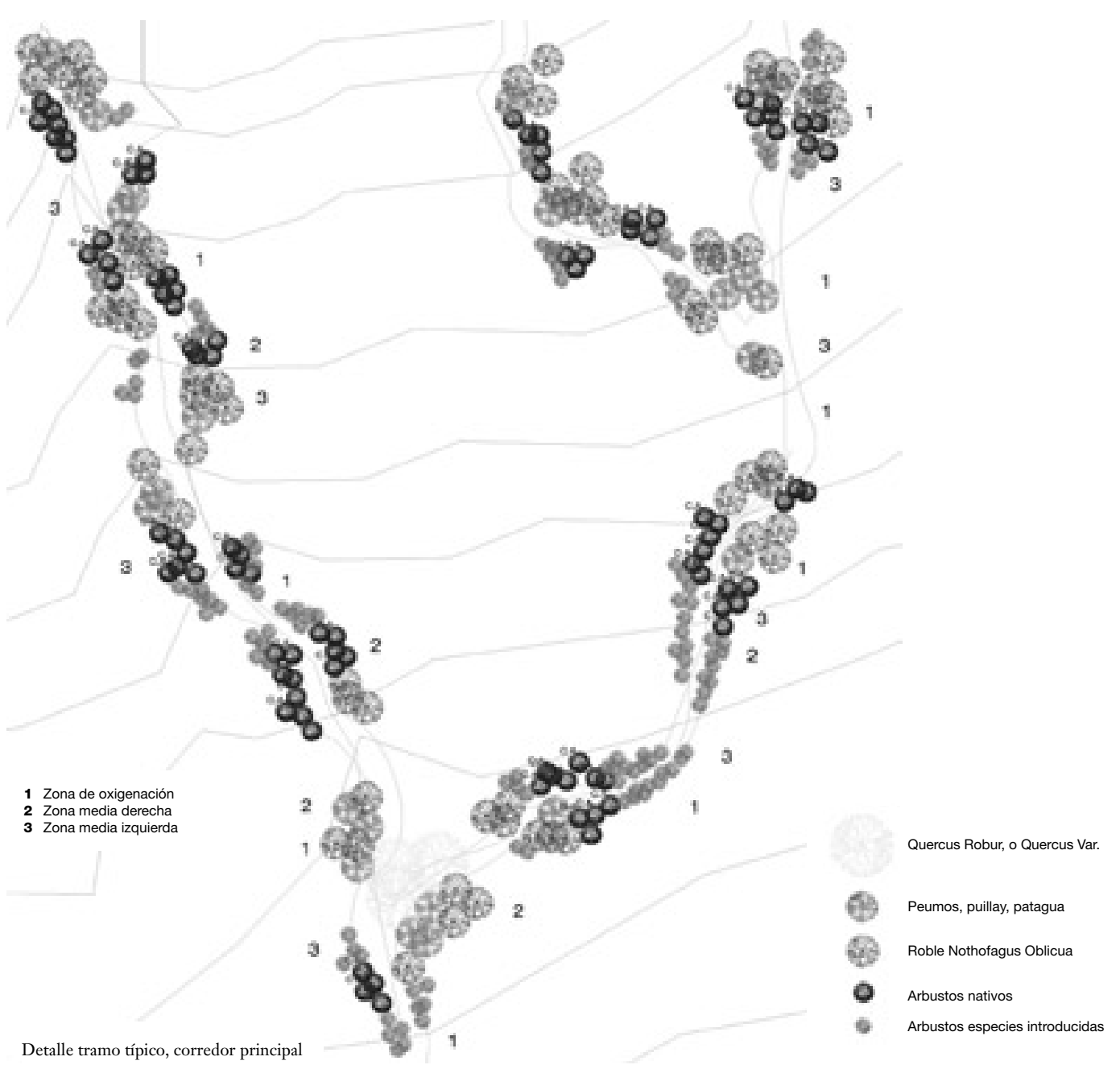

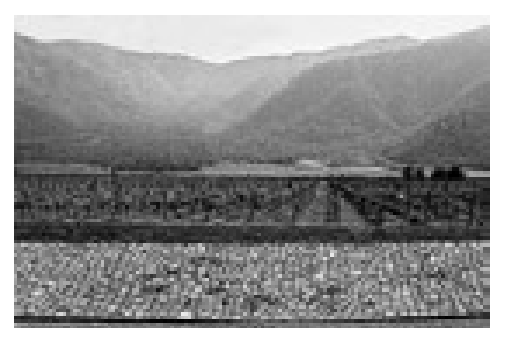

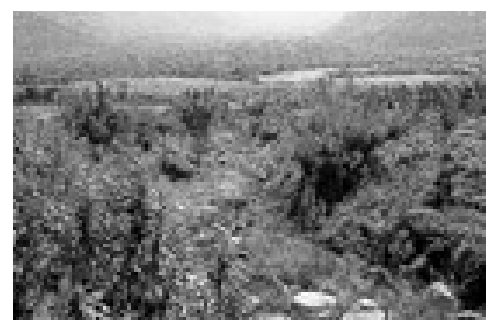

naturales de los cerros al norte, con los espacios cultivados alrededor del río al sur.

\section{Los corredores ecológicos}

En los terrenos agrícolas, al igual que en los medios urbanos y en todos aquellos lugares en que se interviene la naturaleza, el proceso de las aguas, proveniente de la lluvia, pasa a ser un tema de diseño.

Michael Hough define tres factores para el tratamiento del agua en suelos urbanos: las bases ecológicas y funcionales que definen la forma, la integración de objetivos, y la visibilidad de los fenómenos -es decir, transformar la infraestructura en estructura-.

El comportamiento natural del agua se contradice con todas las prácticas de urbanización que básicamente consisten en sacarla lo más rápido posible, haciéndola fluir a altas velocidades por canales, tubos y alcantarillas.

La lección básica de la naturaleza en el ciclo del agua es el almacenamiento. Los pantanos, lagos y napas subterráneas son almacenamientos de reserva de los ríos, reducen la magnitud de las inundaciones río abajo, extendiendo e igualando los flujos en períodos más largos de tiempo. Gertrude Jekill decía "delante de un pantano, arrodíllese". Demás está recordar la capacidad del agua de reunir y generar vida en torno suyo, y la alta capacidad de los pantanos de reciclar desechos.

Además el agua tiene significados profundamente arraigados en nuestra cultura: su contemplación produce fascinación. Integrar los elementos de la naturaleza y su contemplación en el quehacer diario de una explotación agraria es parte esencial de una agricultura biodinámica.

\section{El proyecto en el fundo Los Robles}

Para la viña en el fundo Los Robles se han definido tres corredores, uno central y dos laterales, coincidentes con los espacios por los que naturalmente baja el agua desde los cerros del norte, hasta el río Tinguiririca ubicado al sur. Para todos los corredores se han planteado situaciones para la topografía, el agua y la vegetación. La topografía es la base de los trabajos: define el modo en que escurrirá el agua, los suelos y sus pendientes para las plantaciones y la penetración del viento y el sol.

\section{El agua}

Es importante que los cauces sean protegidos y el agua no se deslice por la pendiente en forma homogénea, recta y rápida. El agua ha de fluir
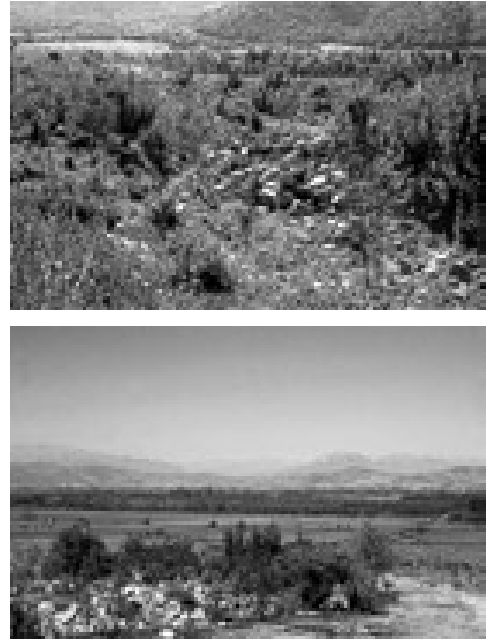

como en la naturaleza, en forma cambiante, a veces rápida, dando saltos, y otras reposada y lenta, apozándose. Se propone una topografía que alterne las situaciones en que el agua corra con rapidez, salte y se arremoline, llamadas zonas de oxigenación, y otras en que se detenga y fluya con calma, llamadas zonas digestoras. Estas zonas se alternarán en forma sucesiva.

Las zonas más estrechas tienen taludes laterales empinados, con el fondo y los laterales protegidos por rocas que el agua no pueda mover, formando pequeños diques. Las zonas en que se pretende que el agua se calme tienen los taludes laterales con una inclinación más suave y las orillas estarán protegidas por vegetación. En plantas y cortes esquemáticos se indican siete diferentes situaciones relacionadas con el tamaño del ancho del cauce.

\section{La vegetación}

Una hacienda biodinámica tiende a constituirse en una unidad cerrada. Los corredores y otros lugares no destinados al cultivo principal han de poseer la mayor diversidad biológica posible, lugar para que las plantas nativas se expresen, cultivar plantas para los preparados y el compost e incluso un lugar para las denominadas malezas. En los corredores se propone la plantación de 
a- Zona oxigenación

El cauce ex expinados $d$ modo que el agua corra con rapidez. El cauce ha de la erosión. A ambos lados delr perennifolios uniendo las dos orillas del cauce creando un túnel sombrío sobre el agua

b- Zona media derecha

政 El borde derecho se planta con árboles y arbustos, borde izquierdo tiene arbustos grandes. Se establece y la entrada del sol

c- Zona media izquierda

El borde izquierdo tiene un talud más suave, cercano erecho es empinado y enrocado. especies menores. árboles caducifolios. De esta manera se crea un

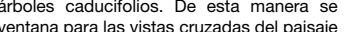

d- Zona media

Ambos bordes tienen taludes cercanos a $30^{\circ}$ creando un espacio más abierto. Uno de los bordes tiene árboles, el otro tiene arbustos y plantas menores. Se
crea un espacio asoleado que relaciona las dos orillas

e- Zona ancha digestora derecha El borde derecho tiene un talud muy suave relacionado con la superficie superior. El borde izquierdo es empinado y enrocado. El borde derecho tiene plantas borde izquierdo es plantado la superticie superior

f - Zona ancha digestora izquierda El borde izquierdo tiene un talud muy suave relacionado empinado y enrocado. El borde izquierdo tiene planta menores relacionadas con las plantaciones en árboles caducifolios

g- Zona ancha digestora

Ambos bordes tienen taludes muy suaves creando un espacio abierto relacionado con los bordes. Ambos bolacion con
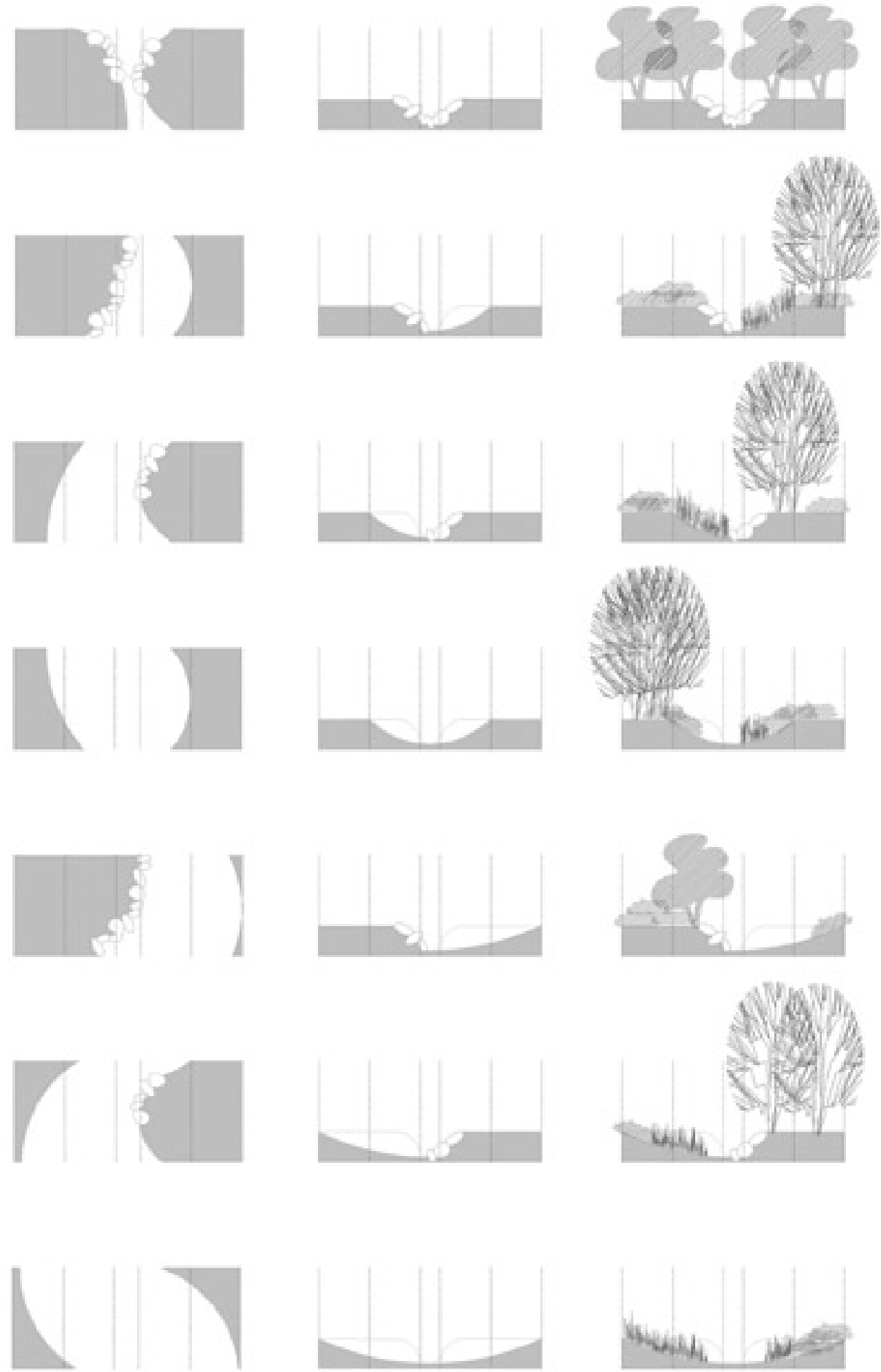
árboles y arbustos y perennes relacionándolos con las situaciones topográficas propuestas para los cauces del agua.

En general se plantarán árboles cercanos a los cauces en las orillas más empinadas, de modo que se cree una situación serpenteante en tres dimensiones. Así, las zonas de oxigenación llevarán árboles en ambas riberas, en las zonas medias se plantarán en la vertiente más empinada y en las zonas anchas se dejarán para la plantación de especies menores. En general se plantarán más árboles en las zonas altas con una proporción de árboles nativos mayor; en las zonas bajas se plantarán menos árboles y predominarán las especies introducidas. Es importante considerar que no es necesario ni aconsejable plantar todos los árboles al mismo tiempo o en una temporada, el sistema se ha de implementar con el tiempo. También es importante señalar que se alternarán árboles nativos con especies introducidas, incluyendo las especies frutales o de huerto.

Los árboles corresponden a especies que existen en los cerros cercanos, como robles, nothofagus oblicua var. macrocarpa, peumos, pataguas, lingues, quillayes, boldos, pelú- pelus, maitenes y sauces chilenos. Las especies introducidas utilizadas son principalmente quercus robur, necesarios para la elaboración de preparados biodinámicos, otras variedades de quercus y olivos; frutales como manzanos, ciruelos y cítricos.

Se emplearon arbustos como arrayanes, corcolenes, molles, colliguayes, mayus y ñipas; especies perennes y arbustos pequeños como michayes y otros berberis, matico, ruda, lupinos, alcaparras, quebrachos, huilmos, e introducidos como lavandas, romero, malvas, tomillo $\mathrm{y}$ hebes.

Se deben cuidar, distribuir y plantar en la medida de lo posible aquellas plantas beneficiosas tales como mentas, manzanillas, dedales de oro, cardos, milenrama, hinojo, eneldo, valeriana, y leguminosas como alfalfa y guisantes -que sirven para fijar minerales y microelementos, además de constituirse en nichos para insectos beneficiosos como abejas y chinitas.

Los espacios de los corredores han de integrarse como un conjunto con todos aquellos otros elementos necesarios para la explotación y manutención de la actividad agrícola, como recorridos, cercas, puertas, accesos, casetas de bombas, lugares de almacenamiento, bodegas de herramientas, y con aquellos destinados a visitantes: hitos, miradores, sombreaderos, patios con sus cercas, muros, vallas y senderos. ARQ

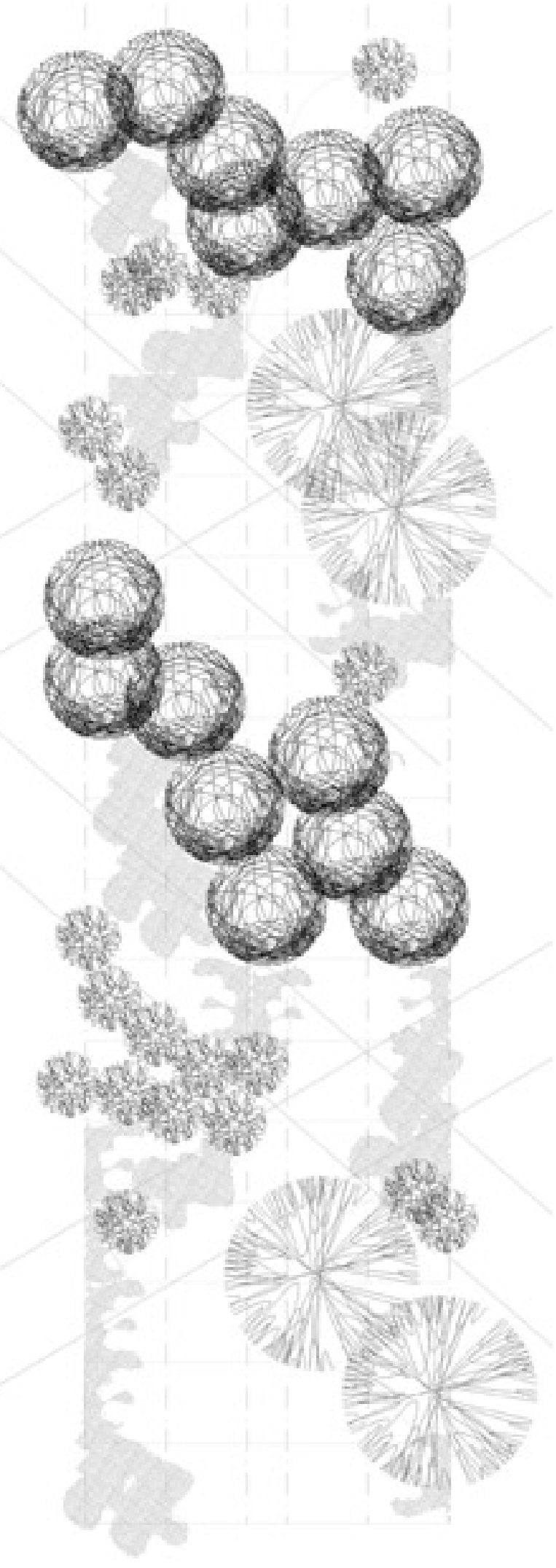

Planta esquemática corredor: Los corredores han de conformarse en una simbiosis entre la topografía, el agua y la vegetación
El agua

El agua se trata como un rí serpenteante, al igual que en la naturaleza, difierente a un canal recto y para distintos tipos de escurrimiento del agua. Han de haber zonas angostas, embudos o zonas de oxigenación, en las cuales el agua corra rápido y salte, alternadas con zonas mas anchas, llamadas agua se calme

Topografía

En directa relación con el agua, los bordes del cauce serán más pronunciados o verticales, en las zonas cercanas; en cambio serán suaves y con forma de playa en las zonas digestoras. En estas zonas pendientes de cada orilla se han de ir alternando. Se indican siete tipos de cortes para producir diferentes situaciones

getación

La vegetación se propone relacionada con el agua y oxigenación, se proponen árboles perennifolios

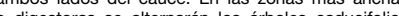
o digestoras se alternaran los árboles caducifolios la vegetación al mismo tiempo que estratificarse por pisos se irá alternando a lo largo del desarrollo de los corredores. Las diferentes ventanas que este sistem produce relaciona los condores y los disentes sectores en un total lo más diverso posible

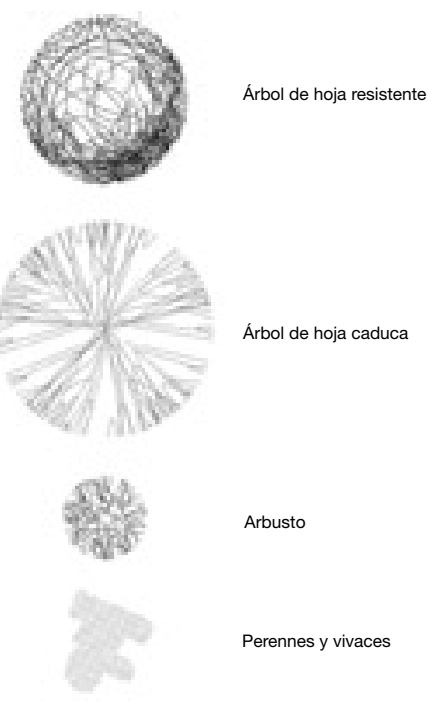

Corredores ecológicos

Arquitecto Teodoro Fernández

Arquitecta asociada Paulina Courard Ubicación Fundo Los Robles, Palmilla, San Fernando, Chile

Mandante Viñedos orgánicas Emiliana Construcción Misuel Elisald

Especies empleadas Notofagus oblicua, peumos, quillayes, piedras del lusar Superficie terreno 120 ha

Año proyecto 2001

Año construcción En ejecución

Fotografías Teodoro Fernández 